\title{
On the derivatives of bivariate Fibonacci polynomials
}

\section{Tuba Çakmak and Erdal Karaduman}

\author{
Department of Mathematics, Faculty of Science \\ Atatürk University, Turkey \\ e-mails: cakmaktuba@yahoo.com, \\ eduman@atauni.edu.tr
}

Received: 8 April $2018 \quad$ Revised: 5 September $2018 \quad$ Accepted: 13 September 2018

\begin{abstract}
In this study, the new algebraic properties related to bivariate Fibonacci polynomials have been given. We present the partial derivatives of these polynomials in the form of convolution of bivariate Fibonacci polynomials. Also, we define a new recurrence relation for the $r$-th partial derivative sequence of bivariate Fibonacci polynomials.
\end{abstract}

Keywords: $k$-Fibonacci sequences, Bivariate Fibonacci polynomials, Partial derivatives of bivariate Fibonacci polynomials.

2010 Mathematics Subject Classification: 11B39, 11B83, 26A24.

\section{Introduction}

In modern science, there is a huge interest in the theory and application of the Golden Section and Fibonacci numbers [1-17]. The Fibonacci numbers $F_{n}$ are the terms of the sequence $0,1,1,2,5, \ldots$, where $F_{n}=F_{n-1}+F_{n-2}, n \geq 2$, with the initial values $F_{0}=0$ and $F_{1}=1$. Falcon and Plaza [2] introduced a general Fibonacci sequence that generalizes the classical Fibonacci sequence. These general $k$-Fibonacci numbers $F_{k, n}$ are defined by $F_{k, n}=k F_{k, n-1}+F_{k, n-2}, n \geq 2$, with the initial conditions $F_{k, 0}=0$ and $F_{k, 1}=1$. If $k$ is a real variable, then $F_{k, n}$ will be equal to $F_{x, n}$ and they correspond to Fibonacci polynomials.

Fibonacci polynomials were studied in 1883 by the Belgian mathematician Eugene Charles and German mathematician E. Jacobsthal. The polynomials $F_{n}(x)$ were defined by the recurrence 
relation

$$
F_{n}(x)=x F_{n-1}(x)+F_{n-2}(x), \quad n \geq 2
$$

where $F_{0}(x)=0$ and $F_{1}(x)=1$. The Fibonacci polynomials and their relationship to diagonals of Pascal's triangle were generalized by Hoggat and Bicknell [5]. Some relationships between Zeckendorf theorem and Fibonacci polynomials [6] were examined. Their divisibility properties had been found by Jacob et al. [10] and Webb and Parberry [17], Hoggat and Bicknell [7] found the roots of Fibonacci polynomials of degree $n$. In [11], $h(x)$-Fibonacci polynomials were defined that generalize both Catalan's and Bryd's Fibonacci polynomials. In [2], Falcon and Plaza investigated the derivatives of these polynomials and they had given many relations for the derivatives of Fibonacci polynomials.

Afterwards, some new generalizations were identified about Fibonacci polynomials which were given by Catalan. One of them is bivariate Fibonacci polynomials, defined as

$$
F_{n}(x, y)=x F_{n-1}(x, y)+y F_{n-2}(x, y), \quad n \geq 2 .
$$

Many properties for selected values of the variables and some recurrence relations of bivariate Fibonacci and Lucas polynomials were obtained [1]. In [9], the properties of bivariate Fibonacci polynomials order $k$ had been investigated in terms of the generating functions. M. N. S. Swamy [12] derived some new properties concerning the derivatives of bivariate Fibonacci and Lucas polynomials. In [3, 4] the works of Filipponi and Horadam revealed the first and second order derivative sequences of Fibonacci and Lucas polynomials and these results had been extended to the $k$-th derivative case as conjectured in [4] and then had been confirmed in [16]. Filipponi and Horadam [4] considered the partial derivative sequences of bivariate second order recurrence polynomials. In [15], Yu and Liang extended some of the results and derived some identities involving the partial derivative sequences of bivariate Fibonacci and Lucas polynomials. One of the generalization of Fibonacci polynomials is given by Tuğlu, Koçer and Stakhov [13]. Also in [14], Claudio de Jesus Pita Ruiz Velasco defines bivariate $s$-Fibopolynomials and gives some of the derivative identities.

This paper is based on the definition of Falcon and Plaza [2] and Tuğlu, Koçer, Stakhov [13]. We study on the derivatives of bivariate Fibonacci polynomials in the form of convolution of these polynomials. In this sense, the present paper is organised as follows. In Section 2, a brief summary of the previous results obtained by Falcon and Plaza in [2] is given. In Section 3, some basic facts are given about bivariate Fibonacci Polynomials. Section 4 presents some new relations related with the derivatives of bivariate Fibonacci Polynomials and gives a new recurrence relation for the $r$-th partial derivative sequence.

\section{The Fibonacci polynomials}

In this section, we will give some basic facts related to Fibonacci polynomials. These results and more can be found in [2].

The $k$-Fibonacci sequence, namely $\left\{F_{k, n}\right\}_{n \in \mathbb{N}}$ has been defined recurrently by $F_{k, n+1}=$ $k F_{k, n}+F_{k, n-1}$ for $n \geq 1$ and any positive real number $k$, with initial conditions $F_{k, 0}=0, F_{k, 1}=1$. 
If $k$ is a real variable $x$ then $F_{k, n}=F_{x, n}$ and they correspond to the Fibonacci polynomials. The Fibonacci polynomials are defined as follows

$$
F_{n+1}(x)=\left\{\begin{array}{ll}
1, & n=0 \\
x, & n=1 \\
x F_{n}(x)+F_{n-1}(x), & n \geq 2
\end{array} .\right.
$$

According to this definition, the sequence of the Fibonacci polynomials is

$$
\left\{F_{n}(x)\right\}=\left\{1, x, x^{2}+1, x^{3}+2 x, x^{4}+3 x^{2}+1, \ldots\right\} .
$$

Note that the $k$-Fibonacci polynomials are the natural extension of the $k$-Fibonacci numbers. The general term of the Fibonacci polynomials is

$$
F_{n+1}(x)=\sum_{i=0}^{\left\lfloor\frac{n}{2}\right\rfloor}\left(\begin{array}{c}
n-i \\
i
\end{array}\right) x^{n-2 i}, \quad n \geq 0 .
$$

On the other hand, by deriving the elements of the sequence of Fibonacci polynomials the following derivative sequence is obtained:

$$
\left\{F_{n}^{\prime}(x)\right\}=\left\{0,1,2 x, 3 x^{2}+2,4 x^{3}+6 x, \ldots\right\} .
$$

The general term of the derivative sequence of Fibonacci polynomials is given as

$$
F_{n+1}^{\prime}(x)=\sum_{i=0}^{\left\lfloor\frac{n-1}{2}\right\rfloor}\left(\begin{array}{c}
n-i \\
i
\end{array}\right)(n-2 i) x^{n-1-2 i}, n \geq 1
$$

by deriving equation 2.2 where $F_{1}^{\prime}(x)=0$.

\section{Bivariate Fibonacci polynomials}

One of the generalizations of the Fibonacci-type polynomials and also Fibonacci numbers are bivariate Fibonacci polynomials. The generalized bivariate Fibonacci polynomials are defined as

$$
H_{n}(x, y)=x H_{n-1}(x, y)+y H_{n-2}(x, y), \quad n \geq 2 .
$$

with initial conditions $H_{0}(x, y)=a_{0}, H_{1}(x, y)=a_{1}$ and it is assumed $y \neq 0$ and $x^{2}+4 y \neq 0$. In [1], by taking $a_{0}=0, a_{1}=1$ the bivariate Fibonacci polynomials $F_{n}(x, y)$ and by taking $a_{0}=2$, $a_{1}=x$ the bivariate Lucas polynomials $L_{n}(x, y)$ were obtained, where,

$$
F_{n}(x, y)=x F_{n-1}(x, y)+y F_{n-2}(x, y), \quad n \geq 2
$$

and

$$
L_{n}(x, y)=x_{n-1} L(x, y)+y L_{n-2}(x, y), \quad n \geq 2 .
$$

So, the first bivariate Fibonacci polynomials are

$$
\left\{F_{n}(x, y)\right\}=\left\{0,1, x, x^{2}+y, x^{3}+2 x y, x^{4}+3 x^{2} y+y^{2}, \ldots\right\}
$$


In [8], the general term of bivariate Fibonacci polynomials has been given by

$$
F_{n+1}(x, y)=\sum_{i=0}^{\left\lfloor\frac{n}{2}\right\rfloor}\left(\begin{array}{c}
n-i \\
i
\end{array}\right) x^{n-2 i} y^{i}, \quad n \geq 0 .
$$

Some of relations between bivariate Fibonacci and Lucas polynomials as follows:

- The following equation appear in [12, eq. (2.8)] for all $n \in \mathbb{Z}$,

$$
L_{n}(x, y)=F_{n+1}(x, y)+y F_{n-1}(x, y),
$$

- The following equation appear in [12, eq. (2.11)] for all $n \in \mathbb{Z}$,

$$
\left(x^{2}+4 y\right) F_{n}(x, y)=L_{n+1}(x, y)+y L_{n-1}(x, y)
$$

- The following equation can be seen in [12, eq. (3.10)]

$$
\frac{\partial L_{n}(x, y)}{\partial x}=n F_{n}(x, y)
$$

\section{Expression of the derivative of bivariate Fibonacci polynomials}

In this section, we establish many formulas and relations for the derivatives of the bivariate Fibonacci polynomials. Their derivatives are given as convolution of bivariate Fibonacci polynomials. This fact allows us to present a family of integer sequences in a new and direct way. Also, we give a new recurrence relation for the $r$-th partial derivative sequence.

If the equation 3.4 differantiate with respect to $x$ and $y$ the folllowing equalities are obtained

$$
\begin{gathered}
\frac{\partial F_{n+1}(x, y)}{\partial x}=\sum_{i=0}^{\left\lfloor\frac{n-1}{2}\right\rfloor}\left(\begin{array}{c}
n-i \\
i
\end{array}\right)(n-2 i) x^{n-1-2 i} y^{i}, \quad n \geq 1 \\
\frac{\partial F_{n+1}(x, y)}{\partial y}=\sum_{i=0}^{\left\lfloor\frac{n-1}{2}\right\rfloor}\left(\begin{array}{c}
n-i \\
i
\end{array}\right)(i) x^{n-2 i} y^{i-1}, \quad n \geq 1
\end{gathered}
$$

where $\frac{\partial F_{1}(x, y)}{\partial x}=\frac{\partial F_{1}(x, y)}{\partial y}=0$ and by [12, eq. (3.12)]

$$
\frac{\partial F_{n}(x, y)}{\partial x}=\frac{\partial F_{n+1}(x, y)}{\partial y}
$$

is written.

The following theorem is a special form of [15, Theorem (2.a)].

Theorem 4.1. If $\frac{\partial F_{1}(x, y)}{\partial x}=0$, for $n>1$, then

$$
\frac{\partial F_{n}(x, y)}{\partial x}=\sum_{i=1}^{n-1} F_{i}(x, y) F_{n-i}(x, y) .
$$


Proof. We prove this conclusion by induction. For $n=2$ it is trivial, since

$$
\sum_{i=1}^{2-1} F_{i}(x, y) F_{2-i}(x, y)=F_{1}(x, y) F_{1}(x, y)=1=\frac{\partial}{\partial x}(x)=\frac{\partial F_{2}(x, y)}{\partial x} .
$$

Let us suppose that the formula is true for $k \leq n$. Then

$$
\begin{aligned}
\frac{\partial F_{n-1}(x, y)}{\partial x} & =\sum_{i=1}^{n-2} F_{i}(x, y) F_{n-1-i}(x, y) \\
\frac{\partial F_{n}(x, y)}{\partial x} & =\sum_{i=1}^{n-1} F_{i}(x, y) F_{n-i}(x, y) .
\end{aligned}
$$

By deriving the equation 3.2 according to variable $x$ and using previous expression we get

$$
\begin{aligned}
\frac{\partial F_{n+1}(x, y)}{\partial x} & =F_{n}(x, y)+x \frac{\partial F_{n}(x, y)}{\partial x}+y \frac{\partial F_{n-1}(x, y)}{\partial x} \\
& =F_{n}(x, y)+x\left(\sum_{i=1}^{n-1} F_{i}(x, y) F_{n-i}(x, y)\right)+y\left(\sum_{i=1}^{n-2} F_{i}(x, y) F_{n-1-i}(x, y)\right) \\
& =F_{n}(x, y)+x F_{1}(x, y) F_{n-1}(x, y)+\sum_{i=1}^{n-1} F_{i}(x, y)\left[x F_{n-i}(x, y)+y F_{n-1-i}(x, y)\right] \\
& =F_{n}(x, y) F_{1}(x, y)+F_{n-1}(x, y) F_{2}(x, y)+\sum_{i=1}^{n-2} F_{i}(x, y) F_{n-i+1}(x, y) \\
& =\sum_{i=1}^{n} F_{i}(x, y) F_{n-i+1}(x, y) .
\end{aligned}
$$

Corollary 4.1.1. For $n>1$

$$
\frac{\partial F_{n}(x, y)}{\partial y}=\sum_{i=1}^{n-2} F_{i}(x, y) F_{n-1-i}(x, y)
$$

where $\frac{\partial F_{1}(x, y)}{\partial x}=0$.

\section{Theorem 4.2.}

$$
\frac{\partial F_{n+1}(x, y)}{\partial x}=\sum_{i=0}^{\left\lfloor\frac{n-1}{2}\right\rfloor}(-1)^{i}(n-2 i) F_{n-2 i}(x, y) y^{i}, \quad n \geq 1 .
$$

Proof. We proof this by induction. It is clear that the claim is true for $n=1$. Let us suppose that the claim is true for $n$. If $n$ is an even integer, by induction hypothesis we get

$$
\frac{\partial F_{2 p+1}(x, y)}{\partial x}=\sum_{i=0}^{p-1}(-1)^{i}(2 p-2 i) F_{2 p-2 i}(x, y) y^{i}
$$


and

$$
\begin{aligned}
\frac{\partial F_{2 p}(x, y)}{\partial x} & =\sum_{i=0}^{p-1}(-1)^{i}(2 p-1-2 i) F_{2 p-1-2 i}(x, y) y^{i} \\
& =\sum_{i=0}^{p-1}(-1)^{i}(2 p-2 i) F_{2 p-1-2 i}(x, y) y^{i}+\sum_{i=0}^{p-1}(-1)^{1+i}(2 p-1-2 i) y^{i} .
\end{aligned}
$$

Now, we will show the equation is true for $n+1$ namely $2 p+1$. By using 3.2 we get

$$
F_{2 p+2}(x, y)=x F_{2 p+1}(x, y)+y F_{2 p}(x, y) .
$$

Then,

$$
\begin{aligned}
\frac{\partial F_{2 p+2}(x, y)}{\partial x}= & F_{2 p+1}(x, y)+x \frac{\partial F_{2 p+1}(x, y)}{\partial x}+y \frac{\partial F_{2 p}(x, y)}{\partial x} \\
= & F_{2 p+1}(x, y)+x \sum_{i=0}^{p-1}(-1)^{i}(2 p-2 i) y^{i}\left[x F_{2 p-2 i}(x, y)+y F_{2 p-1-2 i}(x, y)\right] \\
& +y \sum_{i=0}^{p-1}(-1)^{1+i}(2 p-1-2 i) y^{i} \\
= & (2 p+1) F_{2 p+1}(x, y)-p(2 p-1) F_{2 p-1}(x, y)+\ldots+(-1)^{p} F_{1}(x, y) y^{p} \\
= & \sum_{i=0}^{p}(-1)^{i}(2 p+1-2 i) F_{2 p+1-2 i}(x, y) y^{i} \\
& \left\lfloor\sum_{i=0}^{\left.\frac{n-1}{2}\right\rfloor}(-1)^{i}(n-2 i) F_{n-2 i}(x, y) y^{i}=\frac{\partial F_{n+1}(x, y)}{\partial x} .\right.
\end{aligned}
$$

The similar proof can be given for the case when $n$ is an odd integer. Thus, the result follows for all natural numbers.

Corollary 4.2.1. For $n \geq 3$

$$
\frac{\partial F_{n}(x, y)}{\partial y}=\sum_{i=0}^{\left\lfloor\frac{n-3}{2}\right\rfloor}(n-2-2 i) F_{n-2-2 i}(x, y) y^{i} .
$$

Theorem 4.3.

$$
F_{n}(x, y)=\frac{1}{n}\left[\frac{\partial}{\partial x} F_{n+1}(x, y)+y \frac{\partial}{\partial x} F_{n-1}(x, y)\right] .
$$

Proof. By using equation 3.2 it can be written

$$
F_{n+1}(x, y)=\sum_{i=0}^{\left\lfloor\frac{n}{2}\right\rfloor}\left(\begin{array}{c}
n-i \\
i
\end{array}\right)(x)^{n-2 i} y^{i}, \quad n \geq 1
$$

and

$$
F_{n-1}(x, y)=\sum_{i=0}^{\left\lfloor\frac{n-2}{2}\right\rfloor}\left(\begin{array}{c}
n-2-i \\
i
\end{array}\right)(x)^{n-2-2 i} y^{i}, \quad n \geq 3 .
$$


Then

$$
\begin{aligned}
F_{n+1}(x, y)+y F_{n-1}(x, y) & =\sum_{i=0}^{\left\lfloor\frac{n}{2}\right\rfloor}\left(\begin{array}{c}
n-i \\
i
\end{array}\right)(x)^{n-2 i} y^{i}+y \sum_{i=0}^{\left\lfloor\frac{n-2}{2}\right\rfloor}\left(\begin{array}{c}
n-2-i \\
i
\end{array}\right)(x)^{n-2-2 i} y^{i} \\
& =x^{n}+\sum_{i=1}^{\left\lfloor\frac{n}{2}\right\rfloor}\left(\begin{array}{c}
n-i \\
i
\end{array}\right)(x)^{n-2 i} y^{i}+\sum_{i=0}^{\left\lfloor\frac{n-2}{2}\right\rfloor}\left(\begin{array}{c}
n-2-i \\
i
\end{array}\right)(x)^{n-2-2 i} y^{i+1} \\
& =x^{n}+\sum_{i=1}^{\left\lfloor\frac{n}{2}\right\rfloor}\left(\begin{array}{c}
n-i \\
i
\end{array}\right)(x)^{n-2 i} y^{i}+\sum_{i=1}^{\left\lfloor\frac{n-2}{2}\right\rfloor}\left(\begin{array}{c}
n-1-i \\
i-1
\end{array}\right)(x)^{n-2-2 i} y^{i} \\
& =x^{n}+\sum_{i=1}^{\left\lfloor\frac{n}{2}\right\rfloor}\left[\left(\begin{array}{c}
n-i \\
i
\end{array}\right)+\left(\begin{array}{c}
n-1-i \\
i-1
\end{array}\right)\right](x)^{n-2 i} y^{i} \\
& =x^{n}+n \sum_{i=1}^{\left\lfloor\frac{n}{2}\right\rfloor}\left(\begin{array}{c}
n-1-i \\
i-1
\end{array}\right) \frac{1}{i}(x)^{n-2 i} y^{i} .
\end{aligned}
$$

Now by deriving the last form, we get

$$
\frac{\partial}{\partial x} F_{n+1}(x, y)+y \frac{\partial}{\partial x} F_{n-1}(x, y)=n x^{n-1}+n \sum_{i=1}^{\left\lfloor\frac{n}{2}\right\rfloor}\left(\begin{array}{c}
n-1-i \\
i-1
\end{array}\right) \frac{n-2 i}{i}(x)^{n-1-2 i} y^{i} .
$$

Then,

$$
\begin{aligned}
\frac{1}{n}\left[\frac{\partial}{\partial x} F_{n+1}(x, y)+y \frac{\partial}{\partial x} F_{n-1}(x, y)\right] & =x^{n-1}+\sum_{i=1}^{\left\lfloor\frac{n}{2}\right\rfloor}\left(\begin{array}{c}
n-1-i \\
i-1
\end{array}\right) \frac{n-2 i}{i}(x)^{n-1-2 i} y^{i} \\
& =\sum_{i=0}^{\left\lfloor\frac{n-1}{2}\right\rfloor}\left(\begin{array}{c}
n-1-i \\
i
\end{array}\right)(x)^{n-1-2 i} y^{i}=F_{n}(x, y)
\end{aligned}
$$

So we are done.

\section{Theorem 4.4.}

$$
\frac{\partial F_{n}(x, y)}{\partial x}=\frac{(n+1) F_{n+1}(x, y)+y(n-1) F_{n-1}(x, y)-2 x F_{n}(x, y)}{x^{2}+4 y}, \quad n \geq 1 .
$$

Proof. By deriving equation 3.6 according to variable $x$ we get

$$
2 x F_{n}(x, y)+\left(x^{2}+4 y\right) \frac{\partial F_{n}(x, y)}{\partial x}=\frac{\partial L_{n+1}(x, y)}{\partial x}+y \frac{\partial L_{n-1}(x, y)}{\partial x} .
$$

At this point, by using equation 3.6 the conclusion can be seen.

Corollary 4.4.1. For $n>1$

$$
\frac{\partial F_{n}(x, y)}{\partial x}=\frac{(n) F_{n}(x, y)+y(n-2) F_{n-2}(x, y)-2 x F_{n-1}(x, y)}{x^{2}+4 y} .
$$




\section{Theorem 4.5.}

$$
\frac{\partial^{r} F_{n+1}(x, y)}{\partial x^{r}}=\left\{\begin{array}{lc}
\text { For } n<r, & 0 \\
\text { For } n=r, & r ! \\
\text { For } n>r, & \frac{1}{n-r}\left[n x \frac{\partial^{r} F_{n}(x, y)}{\partial x^{r}}+y(n+r) \frac{\partial^{r} F_{n-1}(x, y)}{\partial x^{r}}\right]
\end{array}\right.
$$

Proof. We prove this by induction. For $r=1$, three subcases occur;

- If $n<1$, then $\frac{\partial F_{1}(x, y)}{\partial x}=0$,

- If $n=1$, then $\frac{\partial F_{2}(x, y)}{\partial x}=(x)^{\prime}=1$ !,

- If $n>1$, then by deriving the equation 3.2 according to variable $x$ we get

$$
\frac{\partial F_{n+1}(x, y)}{\partial x}=F_{n}(x, y)+x \frac{\partial F_{n}(x, y)}{\partial x}+y \frac{\partial F_{n-1}(x, y)}{\partial x}
$$

and by Theorem 4.3 the following conclusion is obtained:

$$
\frac{\partial F_{n+1}(x, y)}{\partial x}=\frac{1}{n-1}\left[n x \frac{\partial F_{n}(x, y)}{\partial x}+y(n+1) \frac{\partial F_{n-1}(x, y)}{\partial x}\right]
$$

So, the claim holds for $r=1$. Let us suppose that the equation is true for the $r$-th partial derivative and we will induct on $r$.

- If $n<r+1$, from induction hypothesis we can write $\frac{\partial^{r+1} F_{n+1}(x, y)}{\partial x^{r+1}}=0$,

- If $n=r+1$, by deriving the equation 3.2$)(r+1)$ times according to variable $x$ we get

$$
\begin{aligned}
\frac{\partial^{r+1} F_{n+1}(x, y)}{\partial x^{r+1}} & =(r+1) \frac{\partial^{r} F_{n}(x, y)}{\partial x^{r}}+x \frac{\partial^{r+1} F_{n}(x, y)}{\partial x^{r+1}}+y \frac{\partial^{r+1} F_{n-1}(x, y)}{\partial x^{r+1}} \\
& =(r+1) r !=(r+1) !
\end{aligned}
$$

- If $n>r+1$, by considering the induction hypothesis

$$
\frac{\partial^{r} F_{n+1}(x, y)}{\partial x^{r}}=\frac{1}{n-r}\left[n x \frac{\partial^{r} F_{n}(x, y)}{\partial x^{r}}+y(n+r) \frac{\partial^{r} F_{n-1}(x, y)}{\partial x^{r}}\right] .
$$

If this equation is derived according to variable $x$ we get

$$
\frac{\partial^{r+1} F_{n+1}(x, y)}{\partial x^{r+1}}=\frac{1}{n-r}\left[n \frac{\partial^{r} F_{n}(x, y)}{\partial x^{r}}+n x \frac{\partial^{r+1} F_{n}(x, y)}{\partial x^{r+1}}+y(n+r) \frac{\partial^{r+1} F_{n-1}(x, y)}{\partial x^{r+1}}\right] \text {. }
$$

If the equations 4.4 and 4.5 are considered together, the desired recurrence relation can be obtained:

$$
\frac{\partial^{r+1} F_{n+1}(x, y)}{\partial x^{r+1}}=\frac{1}{n-r-1}\left[n x \frac{\partial^{r+1} F_{n}(x, y)}{\partial x^{r+1}}+y(n+r+1) \frac{\partial^{r+1} F_{n-1}(x, y)}{\partial x^{r+1}}\right]
$$

Corollary 4.5.1. For $n>1$

$$
\frac{\partial^{r} F_{n+1}(x, y)}{\partial x^{r}}= \begin{cases}\text { For } n<r, & 0 \\ \text { For } n=r, & r ! \\ \text { For } n>r, & \frac{1}{n-r-1}\left[(n-1) x \frac{\partial^{r} F_{n-1}(x, y)}{\partial x^{r}}+y(n-1+r) \frac{\partial^{r} F_{n-2}(x, y)}{\partial x^{r}}\right]\end{cases}
$$




\section{Acknowledgements}

The authors thank to the referees for their thorough reading and beneficial suggestions.

\section{References}

[1] Catalani, M. (2004) Some formulae for bivariate Fibonacci and Lucas polynomials. arXiv preprint math/0406323.

[2] Falcon, S., \& Plaza, Á. (2009) On $k$-Fibonacci sequences and polynomials and their derivatives, Chaos, Solitons \& Fractals, 39(3), 1005-1019.

[3] Filipponi, P., \& Horadam, A. F. (1991) Derivative sequences of Fibonacci and Lucas polynomials, In Applications of Fibonacci numbers, 4, 99-108.

[4] Filipponi, P., \& Horadam, A. F. (1996) Partial Derivative Sequences of Second-Order Recurrence Polynomials, In Applications of Fibonacci Numbers, 105-122.

[5] Hoggatt Jr, V. E., \& Bicknell, M. (1973) Generalized Fibonacci polynomials. Fibonacci Quarterly, 11(5), 457-465.

[6] Hoggatt Jr, V. E., \& Bicknell, M. (1973) Generalized Fibonacci Polynomials and Zeckendorf Theorem, The Fibonacci Quarterly, 11(4), 399-419

[7] Hoggatt Jr, V. E., \& Bicknell, M. (1973) Roots of Fibonacci polynomials, The Fibonacci Quarterly, 11(3), 271-274.

[8] Hoggatt Jr, V. E., \& Long, C. T. (1974) Divisibility Properties of Generalized Fibonacci Polynomials. The Fibonacci Quarterly 12(2), 113-120.

[9] Inoue, K., \& Aki, S. (2011) Bivariate Fibonacci polynomials of order $k$ with statistical applications, Annals of the Institute of Statistical Mathematics, 63(1), 197-210.

[10] Jacob, G., Reutenauer, C., \& Sakarovitch, J. (2006) On a divisibility property of Fibonacci polynomials, preprint available at http://en. scientificcommons. org/43936584.G.

[11] Nalli, A., \& Haukkanen, P. (2009) On generalized Fibonacci and Lucas polynomials, Chaos, Solitons \& Fractals, 42(5), 3179-3186.

[12] Swamy, M. N. S. (1999) Generalized Fibonacci and Lucas polynomials, and their associated diagonal polynomials, Fibonacci Quarterly, 37, 213-222.

[13] Tuğlu, Koçer, E. G., \& Stakhov, A. (2011) Bivariate Fibonacci-like p-polynomials, Applied Mathematics and Computations, 217(24), 10239-10246.

[14] Velasco, C. J. P. R. (2012) On bivariate $s$-Fibopolynomials, arXiv preprint:1203.6055. 
[15] Yu, H., \& Liang, C. (1997) Identities involving partial derivatives of bivariate Fibonacci and Lucas polynomials, Fibonacci Quarterly, 35, 19-23.

[16] Wang, J. (1995) On the $k$-th Derivative Sequences of Fibonacci and Lucas Polynomials. The Fibonacci Quarterly, 33.2: 174-178.

[17] Webb, W. A., \& Parberry, E. A. (1969) Divisibility properties of Fibonacci polynomials, Fibonacci Quarterly, 7(5), 457-463. 